\title{
First-line Treatment of Metastatic Lung Cancer-New Developments
}

\author{
Grace K Dy, MD' ${ }^{1}$ and Alex A Adjei, MD, PhD²
}

\begin{abstract}
1. Attending Physician and Assistant Professor of Medicine, Department of Medicine; 2. Senior Vice President of Clinical Research,
\end{abstract} Chair, Department of Medicine, and Katherine Anne Gioia Chair in Cancer Medicine, Roswell Park Cancer Institute

DOI: $10.17925 /$ OHR.2008.04.1.31

Systemic chemotherapy as palliative therapy for patients with advanced non-small-cell lung cancer (NSCLC) not amenable to curative therapy can provide meaningful symptom improvement and survival advantage over best supportive care. ${ }^{1}$ While single agents have demonstrable efficacy and less toxicity, a meta-analysis showed that doublet regimens employing modern cytotoxic agents confer a survival advantage relative to single agents. ${ }^{2}$ Thus, among patients with good performance status, platinum-containing doublets constitute the accepted standard of care for the first-line treatment of advanced NSCLC. ${ }^{3,4}$

\section{Molecular Targeted Therapies}

Epidermal growth factor receptor (EGFR) is a member of the Erb-B family of transmembrane glycoprotein receptors that is aberrantly expressed in up to $80 \%$ of cases of NSCLC, and is associated with worse prognosis. ${ }^{5}$ Erlotinib and gefitinib are both orally active inhibitors of EGFR tyrosine kinase (TK) domain. Whereas neither agent improved survival outcomes in combination with chemotherapy in the first-line setting, ${ }^{6-9}$ both continue to be developed as front-line therapies, either singly or in combination with other molecular targeted therapies. This is based on the striking clinical activity observed among non-smokers, particularly those of Asian ethnicity with adenocarcinoma histology.

In contrast to the oral EGFR TK inhibitors, intriguing results are seen when first-line platinum doublet is combined with cetuximab, a monoclonal antibody against the extracellular domain of EGFR. Two recent phase III trials testing cetuximab with chemotherapy in the first-line setting have been completed. The First-line in Lung cancer with Erbitux (FLEX) trial was a multicenter randomized comparison of cisplatin and vinorelbine with or without cetuximab in 1,125 patients with treatment-naïve stage IIIB/IV and Eastern Co-operative Oncology Group (ECOG) performance status (PS) score of $0-2$ whose tumors expressed EGFR. ${ }^{10}$ Twenty-two percent of patients in each arm were non-smokers, and patients of Caucasian descent accounted for over $80 \%$ of the intake in each arm. The trial was conducted at 166 centers worldwide, including Europe, South America, and Asia. Besides the anticipated statistically significant increased rate of skin rash, diarrhea, and infusion-related reactions in the cetuximab arm, febrile neutropenia was also increased ( 22 versus $15 \% ; p<0.05$ ). The primary endpoint of overall survival (OS) was improved in patients receiving the cetuximab combination (11.3 versus 10.2 months; $p=0.044$ ), with prespecified subgroup analyses suggesting a greater benefit among Caucasians independent of histology. There was no difference in overall progression-free survival (PFS). There was also no OS benefit in the Asian subgroup, although this is likely a reflection of small sample size as well as the availability of effective salvage therapies such as EGFR TK inhibitors.
The other trial, BMS-099, performed in the US and Canada, is a comparison of carboplatin with a taxane with or without cetuximab in 676 patients with advanced NSCLC." Positive EGFR staining was not required. No significant difference was found in the primary end-point of PFS as determined by the Institutional Research Review Committee (IRRC). Response rate, as assessed by the IRRC, and PFS, as assessed by clinical investigators, were statistically significant and favored the cetuximab arm. Pre-specified subset analyses revealed greater improvement in PFS with cetuximab among patients treated with docetaxel (versus paclitaxel) and with ECOG PS 0 (versus higher ECOG PS). Disappointingly, a recent press release of updated survival analysis did not reveal a survival difference with the addition of cetuximab (9.7 versus 8.4 months, hazard ratio $[H R]=0.89 ; p=0.17) .{ }^{12}$ Unlike the FLEX trial, this study was not adequately powered to detect a small improvement in OS; these results in addition to the marginal (four-week) increase in median OS in the FLEX trial suggest that the benefits of adding cetuximab to chemotherapy in an unselected NSCLC population may be small.

The first successful breakthrough in the existing first-line chemotherapy regimens for advanced NSCLC was in combination with bevacizumab, a monoclonal antibody that targets the vascular endothelial growth factor (VEGF). Patients with squamous cell histology were excluded from the pivotal phase III trial, E4599, because of an apparent increased risk for fatal hemoptysis observed in the phase $\|$ trial. ${ }^{13}$ This study randomized

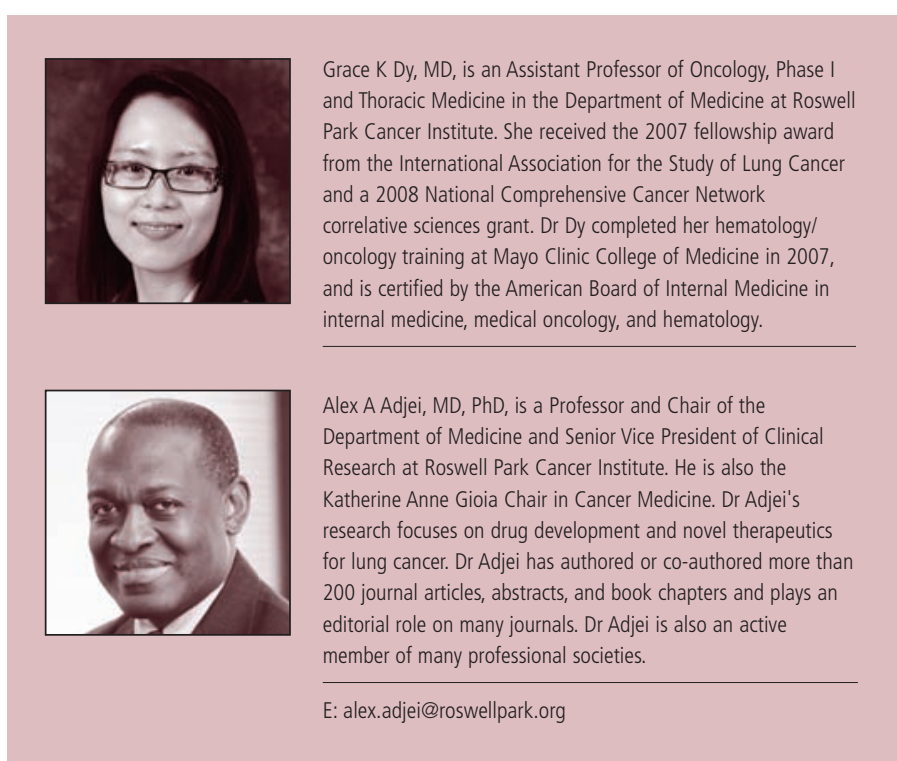




\section{Lung Cancer}

878 patients with non-squamous histological disease to receive six cycles of paclitaxel plus carboplatin with or without bevacizumab. ${ }^{14}$ Bevacizumab was continued until progressive disease or unacceptable toxic effects. Overall survival in patients with advanced NSCLC who received first-line treatment with carboplatin/paclitaxel with bevacizumab (at $15 \mathrm{mg} / \mathrm{kg}$ dose) was improved compared with chemotherapy alone (12.3 versus 10.3 months, respectively, $H R=0.80 ; p=0.003)$. On the other hand, the incidences of grade 3-5 neutropenia, hemorrhage, and treatment-related deaths were higher in patients treated with bevacizumab plus chemotherapy, particularly in the elderly population ( $>70$ years of age), where there was no OS benefit with the addition of bevacizumab. ${ }^{15}$

\section{The first successful breakthrough in}

the existing first-line chemotherapy

regimens for advanced non-small-cell

lung cancer was in combination with

bevacizumab, a monoclonal antibody

that targets the VEGF.

The similarly designed phase III placebo-controlled European Avastin in Lung Cancer (AVAIL) trial combined bevacizumab at two different doses (7.5 and $15 \mathrm{mg} / \mathrm{kg}$ ) with cisplatin and gemcitabine followed by maintenance bevacizumab treatment. The study met its primary endpoint with a median PFS of 6.1 months in the chemotherapy group compared with 6.7 months $(p=0.003)$ and 6.5 months $(p=0.03)$ in the low- and high-dose bevacizumab groups, respectively. ${ }^{16}$ While median survival of patients in all arms of the study exceeded one year, there was no statistically significant difference in OS among the treatment arms. ${ }^{17}$ Despite this, current evidence supports the combination of a platinumbased doublet with bevacizumab as the first-line standard for eligible patients with advanced NSCLC.

Oral inhibitors of VEGF receptor TK have also been evaluated in the treatment of NSCLC. Both sorafenib and sunitinib are multiple kinase inhibitors against VEGF-R, platelet-derived growth factor receptor (PDGFR), C-kit, and Flt-3 that are approved for the treatment of renal cell carcinoma. A randomized phase III study comparing first-line treatment using carboplatin/paclitaxel with or without sorafenib in patients with unresectable NSCLC has been reported. ${ }^{18}$ The study did not meet its primary end-point of improved survival. Moreover, fatal pulmonary hemorrhages predominated in patients with squamous cell histology, and subset analysis suggested a detrimental effect to patients with squamous cell carcinoma who received sorafenib. Based on these results, a similarly designed trial (NEXUS) using gemcitabine and cisplatin as the backbone chemotherapy regimen was amended to exclude patients with squamous histology. Sunitinib is being tested in a randomized phase III placebocontrolled study as a maintenance therapy for patients with NSCLC who have at least stable disease after four cycles of platinum-based chemotherapy, with or without bevacizumab (bevacizumab not to be given beyond the fourth cycle). It is also being studied in two other randomized phase II studies as a first-line treatment together with platinum-based combination with either etoposide or irinotecan for patients with extensive stage small-cell lung cancer. Cediranib (AZD2171) is an oral selective VEGFR TK inhibitor that was being evaluated in a Canadian randomized phase II/III trial (BR24) in combination with paclitaxel/carboplatin as first-line therapy for advanced NSCLC. A planned safety/efficacy interim analysis earlier this year showed an imbalance in toxicity with the cediranib arm, thus the study did not continue into the phase III portion despite evident clinical activity. ${ }^{19}$

Vandetanib (ZD6474) is a dual inhibitor against both VEGFR and EGFR that was evaluated as first-line treatment in 181 patients who were randomized to vandetanib alone, vandetanib plus carboplatin/paclitaxel, or placebo plus carboplatin/paclitaxel..$^{20}$ No significant difference was found in PFS or OS between the chemotherapy alone and the chemotherapy-vandetanib arms, whereas treatment in the vandetanib alone arm was terminated prematurely because of a low PFS. On the other hand, promising activity with improvement in PFS was seen in combination with docetaxel in the second-line setting in a randomized phase II study. ${ }^{21}$ It is currently being tested in a randomized placebocontrolled phase II combination with four cycles of docetaxel plus carboplatin as first-line therapy followed by a maintenance phase. It is also being studied as first-line treatment in extensive-stage SCLC in combination with cisplatin and etoposide, as well as in the neoadjuvant setting for NSCLC in combination with carboplatin and paclitaxel.

Activation of the insulin-like growth factor (IGF) receptor pathway has been implicated in the pathogenesis of multiple malignancies, including lung cancer, ${ }^{22}$ resulting in anti-apoptosis and resistance to anticancer therapies such as cytotoxic chemotherapy, hormonal therapy, molecular targeted agents, and radiation. ${ }^{23-27}$ CP-751,871, a fully humanized monoclonal antibody to IGF-1 receptor, has been tested in a randomized phase II study in combination with chemotherapy using paclitaxel and carboplatin versus chemotherapy alone as first-line treatment for advanced NSCLC. There was an increased response rate among patients receiving CP-751,871, with apparent dose response and a trend toward improved PFS. ${ }^{28}$ Several randomized phase III studies of this agent with chemotherapy and with erlotinib are on the way or have already begun.

\section{Cytotoxic Agents}

Albumin-bound paclitaxel (Abraxane ${ }^{\circledR}$ ) is a Cremophor ${ }^{\circledR}$-free, albuminbound particle form of paclitaxel that has shown promising phase II results in first-line advanced NSCLC. ${ }^{29}$ There is an ongoing registrational phase III trial that will randomize approximately 1,000 patients to carboplatin with albumin-bound paclitaxel or paclitaxel, with response rate as the primary end-point. Emerging data on the histological subtype of NSCLC influencing treatment outcomes have provoked much discussion. We earlier alluded to data showing the safety and efficacy of novel agents in patients with adenocarcinoma. More recently, Scagliotti et al. ${ }^{30}$ reported the results of a non-inferiority phase III trial demonstrating comparable survival outcomes in 1,725 chemotherapynaïve patients with advanced NSCLC randomized to either cisplatin in combination with pemetrexed or cisplatin with gemcitabine. A prespecified analysis of survival by histology showed significantly improved median survival in the pemetrexed-containing arm versus the gemcitabine-containing arm in 847 adenocarcinoma patients (12.6 versus 10.9 months) and in 153 patients with large-cell carcinoma (10.4 versus 6.7 months), whereas a non-significant trend toward better 
survival was found with the gemcitabine combination in 473 patients with squamous cell carcinoma (10.8 versus 9.4 months).

Pemetrexed also took center stage in another placebo-controlled phase III study that evaluated its role as maintenance treatment after first-line chemotherapy compared with placebo. Median PFS showed statistically significant improvement (4.04 versus 1.97 months, HR 0.599; $p<0.00001$ ) with a preliminary analysis showing a trend toward improvement in OS as well with the pemetrexed arm (13.01 versus 10.18 months, HR 0.798; $\mathrm{p}=0.06$ ). Similar to the results reported by Scagliotti et al., differential efficacy was demonstrated when results were stratified according to histology. Outcomes with pemetrexed were significantly improved among patients with non-squamous NSCLC, but not in patients with squamous histology. ${ }^{31}$

Such histological correlations are most likely a crude manifestation of the underlying molecular heterogeity of NSCLC. Higher expression level of thymidylate synthase, a pemetrexed target, in squamous histology has been demonstrated and is a plausible mechanism of relative resistance to pemetrexed. ${ }^{32}$ Similarly, data are emerging showing an direct correlation of ERCC1 gene expression levels with platinum resistance. ${ }^{33}$ A Spanish trial prospectively randomized over 400 patients to a control arm receiving docetaxel plus cisplatin or an experimental genotype-based therapy arm wherein treatment was assigned based on ERCC 1 messenger RNA [mRNA] levels (cislatin/docetaxel for patients with low ERCC1 levels; docetaxel/ gemcitabine for patients with high ERCC 1 levels). ${ }^{34}$ Response rates were statistically higher in the genotypic arm. However, no differences were seen in survival outcomes due to technical and design issues. This study represents a pioneering effort in the establishment of 'molecularly tailored' individualized therapy.

\section{Conclusions}

While platinum-based doublets constitute the backbone of first-line treatment for metastatic NSCLC, recent clinical findings with molecular therapies targeting various signaling pathways have bolstered enthusiasm in this rapidly evolving field. The combination with bevacizumab in appropriately selected patients is the standard in the first-line treatment of patients with advanced NSCLC. Data are also emerging on the clinical utility of combining cetuximab with front-line chemotherapy. In addition, the correlation between treatment efficacy and toxicity of novel molecular targeted therapies and certain cytotoxic agents with histological subtypes of NSCLC represents a simple approach toward therapy tailored to the molecular heterogeneity of NSCLC. Research is ongoing to validate markers of tumor resistance or sensitivity specific to each drug, such as ERCC1 with cisplatin-based regimens. The use of maintenance chemotherapy in the front-line setting has found renewed potential with the availability of effective and well-tolerated agents.
1. Chemotherapy in non-small cell lung cancer: a meta-analysis using updated data on individual patients from 52 randomized clinical trials, Non-small Cell Lung Cancer Collaborative Group, BMJ, 1995;311(7010):899-909.

2. Delbaldo C, Michiels S, Syz N, et al., Benefits of adding a drug to a single-agent or a two-agent chemotherapy regimen in advanced non-small cell lung cancer: a meta-analysis, JAMA 2004;292(4):470-84.

3. Scagliotti GV, De Marinis F, Rinaldi M, et al., Phase III randomized trial comparing three platinum-based doublets in advanced non-small-cell lung cancer, J Clin Oncol, 2002;20:4285-4289.

4. Schiller JH, Harrington D, Belani $\mathrm{CP}$, et al. Comparison of four chemotherapy regimens for advanced non-small-cell lung cancer, N Engl J Med, 2002;346:92-98.

5. Cerny T, Barnes DM, Hasleton $P$, et al., Expression of epidermal growth factor receptor (EGF-R) in human lung tumours, $\mathrm{Br}$ J Cancer, 1986;54(2):265-9.

6. Giaccone G, Herbst RS, Manegold C, et al., Gefitinib in combination with gemcitabine and cisplatin in advanced non-small-cell lung cancer: A phase III trial - INTACT 1, I Clin Oncol, 2004;22:777-84.

7. Herbst RS, Giaccone G, Schiller JH, et al., Gefitinib in combination with paclitaxel and carboplatin in advanced non-small-cell lung cancer: a phase III trial-INTACT 2, J Clin Oncol, 2004;22:785-94.

8. Herbst RS, Prager D, Hermann R, et al., TRIBUTE: a phase III trial of erlotinib hydrochloride (OSI-774) combined with carboplatin and paclitaxel chemotherapy in advanced non-small-cell lung cancer, I Clin Oncol, 2005;23:5892-9.

9. Gatzemeier U, Pluzanska A, Szczesna A, et al., Results of a phase III trial of erlotinib (OSI-774) combined with cisplatin and gemcitabine (GC) chemotherapy in advanced non-small cell lung cancer (NSCLC), I Clin Oncol, 2004:22:7010.

10. Pirker R, Szczesna A, von Pawel J, et al., FLEX: A randomized, multicenter, phase III study of cetuximab in combination with cisplatin/vinorelbine( $\mathrm{CV}$ ) versus $\mathrm{CV}$ alone in the first-line treatment of patients with advanced non-small cell lung cancer (NSCLC), I Clin Oncol, 2008;26: abstract 3.

11. Lynch TJ, Patel T, Dreisbach L, et al., A randomized multicenter phase III study of cetuximab (Erbitux) in combination with Taxane/Carboplatin versus Taxane/carboplatin alone as first line treatment for patients with advanced /metastatic non-small cell lung cancer, J Thoracic Oncol, 2007;2(Suppl. 4):B3-30.
12. IMCLONE Press Release, 2008. Available at: www.istockanalyst. com/article/viewiStockNews+articleid_2562688.html

13. Johnson DH, Fehrenbacher L, Novotny WF, et al., Randomized phase II trial comparing bevacizumab plus carboplatin and paclitaxel with carboplatin and paclitaxel alone in previously untreated locally advanced or metastatic non-small-cell lung cancer, J Clin Oncol, 2004:22:2184-91.

14. Sandler A, Gray R, Perry MC, et al., Paclitaxel - carboplatin alone or with bevacizumab for non-small-cell lung cancer, $N$ Engl J Med, 2006:355:2542-50.

15. Ramalingam SS, Dahlberg SE, Langer CJ, et al., Outcomes for elderly, advanced-stage non small-cell lung cancer patients treated with bevacizumab in combination with carboplatin and paclitaxel: analysis of Eastern Cooperative Oncology Group Trial 4599, J Clin Oncol, 2008;26(1):60-65.

16. Manegold C, von Pawel J, Zatloukal P, et al., Randomised, double-blind multicentre phase III study of bevacizumab in combination with cisplatin and gemcitabine in chemotherapy-naïve patients with advanced or recurrent non-squamous non-small cell lung cancer (NSCLC): B017704, Proc Am Soc Clin Oncol, 2007:25:LBA7514.

17. Genentech press release, 2008. Available at: www.gene.com/ gene/news/press-releases/display.do?method=detail \& id $=11207$

18. Bayer and Onyx press release, 2008. Available at: www.medicalnewstoday.com/articles/105387.php

19. AstraZeneca press release, 2008. Available at: www.astrazenecaus.com/search/?itemld=2289031

20. Heymach J, Paz-Ares L, De Braud F, et al., Randomized phase II study of vandetanib (VAN) alone or in combination with carboplatin and paclitaxel (CP) as first-line treatment for advanced non-small cell lung cancer (NSCLC), Proc Am Soc Clin Oncol, 2007; 25:7544.

21. Heymach JV, Johnson BE, Prager D, et al., Randomized, placebo-controlled phase II study of vandetanib plus docetaxel in previously treated non-small cell lung cancer, I Clin Oncol, 2007;25(27):4270-77.

22. Chang YS, Kong G, Sun S, et al., Clinical significance of insulin-like growth factor-binding protein-3 expression in stage I non-small cell lung cancer, Clin Cancer Res, 2002;8:3796-3802.

23. Allen GW, Saba C, Armstrong EA, et al., Insulin-like growth factor-I receptor signaling blockade combined with radiation, Cancer Res, 2007;67:1155-62.
24. Camirand A, Lu Y, Pollak M, Co-targeting HER2/ErbB2 and insulin-like growth factor-1 receptors causes synergistic inhibition of growth in HER2-overexpressing breast cancer cells, Med Sci Monit, 2002;8:BR521-6

25. Camirand A, Lu Y, Pollak M, Co-targeting HER2/ErbB2 and insulin-like growth factor-1 receptors causes synergistic inhibition of growth in HER2-overexpressing breast cancer cells, Med Sci Monit, 2002;8:BR521-6.

26. Wiseman LR, Johnson MD, Wakeling AE, et al., Type I IGF receptor and acquired tamoxifen resistance in oestrogen-responsive human breast cancer cells, Eur J Cancer, 1993;29A:2256-64.

27. Gooch JL, Van Den Berg CL, Yee D, Insulin-like growth factor (IGF)-I rescues breast cancer cells from chemotherapy-induced cell death - proliferative and anti-apoptotic effects, Breast Cancer Res Treat, 1999;56(1):1-10.

28. Karp DD, Paz-Ares $L G$, Novello $S$, et al., High activity of the anti-IGF-1R antibody CP-751,871 in combination with paclitaxe and carboplatin in squamous NSCLC, I Clin Oncol, 2008:26:8015a

29. Allerton JP, Hagenstad CT, Webb RT, et al., A phase II evaluation of the combination of paclitaxel protein-bound and carboplatin in the first-line treatment of advanced non-small cell lung cancer (NSCLC), Proc Am Soc Clin Oncol, 2006:24(185):7127.

30. Scagliotti GV, Parikh P, von Pawel J, et al., Phase III study comparing cisplatin plus gemcitabine with cisplatin plus pemetrexed in chemotherapy-naive patients with advanced-stage non-small-cell lung cancer, J Clin Oncol, 26:3543-51.

31. Ciuleanu TE, Brodowicz T, Belani CP, et al., Maintenance pemetrexed plus best supportive care (BSC) versus placebo plus BSC: A phase III study, J Clin Oncol, 2008;27(Suppl. 15):426s.

32. Ceppi P, Volante M, Saviozzi S, et al., Squamous cell carcinoma of the lung compared with other isotopes shows higher messenger RNA and protein levels for thymidylate synthase, Cancer, 2006;107:1589-96.

33. Altaha $R$, Liang $X$, Yu JJ, et al., Excision repair cross-complementing-group 1: gene expression and platinum resistance, Int J Mol Med, 2004;14(6):959-70.

34. Cobo M, Isla D, Massuti B, et al., Customizing cisplatin based on quantitative excision repair cross-complementing 1 mRNA expression: A phase III trial in non-small-cell lung cancer, J Clin Oncol, 2007:25:2747-54. 